\title{
Uncertainties Consideration in Empirical Frequency Response Function Data for Damage Identification Based On Artificial Neural Network
}

\author{
Khairul H. Padil ${ }^{1,2 *}$, Norhisham Bakhary ${ }^{1,3}$, Wan Nur Firdaus Wan Hassan ${ }^{1}$, \\ Nadirah Darus ${ }^{1}$
}

${ }^{1}$ School of Civil Engineering, Faculty of Engineering,

Universiti Teknologi Malaysia, 81310 Johor Bahru, Johor, MALAYSIA

${ }^{2}$ Forensic Engineering Centre, Institute for Smart Infrastructure and Innovative Construction,

Universiti Teknologi Malaysia, 81310 Johor Bahru, Johor, MALAYSIA

${ }^{3}$ Institute of Noise and Vibration,

Universiti Teknologi Malaysia, City Campus, 54100 Kuala Lumpur, MALAYSIA

*Corresponding Author

DOI: https://doi.org/10.30880/ijie.2021.13.03.025

Received 05 January 2021; Accepted 13 May 2021; Available online 10 June 2021

\begin{abstract}
The modern application of frequency response function (FRF) with artificial neural networks (ANN) has become one of the leading methods in vibration-based damage detection approach. However, since full-size empirically obtained FRF data is used as ANN input, a broad composition ANN input layer series would occur. Consequently, principal component analysis (PCA) is adopted to compress the FRF data magnitude. Despite this, PCA alone is unable to select the important FRF data features effectively, due to the exceedingly FRF data size in addition with existing uncertainties. Therefore, this study proposed the merger of a non-probabilistic analysis and ANN approach with PCA by considering the uncertainties effect and the inefficiency of using empirical FRF data. The empirical FRF data is obtained from a steel truss bridge structure. The results show that the PoDE values above $95 \%$ are measured at the particular executed damage locations and the DMI values show the damage severity at the actual damage locations. Overall, the results show that the proposed method is capable in considering the uncertainties effect on the empirical FRF data for structural damage identification.
\end{abstract}

Keywords: Frequency response function, uncertainties, non-probabilistic, artificial neural network

\section{Introduction}

Artificial neural networks (ANN) are favoured by many researchers for vibration-based damage detection [1]-[3], as input and output nonlinear relationship parameters can be well established using the method. At the same time, the use of frequency domain data such as frequency response function (FRF) is also preferred [3]-[5], as it offers adequate dynamic features of a structure without the risk of information leakage. For this approach, Principal Component Analysis (PCA) is formulated to compress the FRF data size, as FRF comprises a huge data point's number. Unfortunately, the compressed FRF data is still unable to deliver the exact damage information due to the missing dynamic features, while errors are still introduced into the damage detection procedure. These newly introduced errors causes by PCA process can be categorized as measurement errors [6], [7]. In addition, two principal uncertainties 
including modelling error and measurement noise may intensify these errors effect and lead to inaccurate damage detection.

Modelling error and measurement noise are the uncertainties from finite element (FE) model and real structural testing, respectively. Hence, these three sources of uncertainties including data compression errors, modelling errors and measurement noise are measured as the causes of inaccuracy in ANN predictions using compressed empirical FRF data. Therefore, to counter this drawback of uncertainties in the empirical FRF data, a non-probabilistic analysis with ANN approach is implemented. This implementation of non-probabilistic analysis is proven to be efficient in dealing with uncertainties problem in the modal updating method [8 - 9], modal data [10] and wavelet method [11].

This paper examines the pertinence of the non-probabilistic analysis with ANN approach using compressed modal tested FRF data obtained from an empirical steel truss bridge structure with the consideration of uncertainties for vibration-based damage detection. On this subject, PCA is used to compress the empirically obtained FRF data. Then this compressed FRF data will be selected as a new damage index (DI) for the ANN input variable. Then, the elemental stiffness parameters (ESP) will be used as the ANN output variables. The interval analysis is expressed to quantify the uncertainties and produce the lower and upper bounds of the ANN input (DI) variables and output (ESP) variables. Hence, two ANNs model is designed to classify the uncertainties as upper and lower bounds. Possibility of Damage Existence (PoDE) is molded to localize the damage existence and damage measure index (DMI) is calculated to identify the severity of the damage. A lab scaled steel truss bridge is constructed and modal testing procedure is conducted to obtain the empirical FRF data. Based on the damage detection result of PoDE and DMI, it is exhibited that the pertinence of the non-probabilistic analysis through ANN approach on compressed empirical FRF data manage to identify the damage location and severity.

\section{Compressed FRF Data}

FRF is a complex number (consist of real and imaginary numbers) in term of frequency domain transfer function, which includes the magnitudes and phases of the domain. Hence, the FRF matrix $[H(\omega)]$ is designed as:

$$
[H(\omega)]=\left(-\omega^{2}[M]+i \omega[C]+[K]\right)^{-1}
$$

where $M, C$ and $K$ are mass, damping and stiffness, respectively. This study used the real numbers of FRF complex number to be the ANN input variables founded by Pradhan \& Modak [12].

A new group of PCs which is the impartial variables in the $P$-dimensional space are acquired based on the orthogonal projections then converted from the focal variables of data in the $H$-dimensional space with $P<H$. These PCs are the eigenvectors of the covariance matrix hence categorized as parallel with one another. Reflecting the $H_{(m \times n)}$ with $m$ and $n$ distinct vibration analysis, the loading of the Eigen decomposition can be multiplied correspondingly to create PCs, which given as $\widetilde{H}_{(m \times r)}$. Hence, the DI can be computed as:

$$
\bar{H}_{(n \times r)}=\left[H_{(m \times n)}\right]^{T}\left[\widetilde{H}_{(m \times r)}\right]
$$

\section{Non-Probabilistic ANN}

The ANN is modeled using multilayer perceptron ANN of Levenberg-Marquardt back-propagation algorithm. 20 hidden neurons and tangent sigmoid transfer functions are selected for input, hidden and output layers as described in Fig. 1. As mentioned earlier, DI $(H)$ and ESP $(\alpha)$ are used as the ANN input and output variables respectively. In training phase, random damage cases of the truss structure (by reducing the ESP value) are created using the FE analysis. Meanwhile in testing phase, known elements of damage cases are generated accordingly. Stiffness reduction Factor (SRF) for every segment to characterizes the variations in the stiffness parameter can be expressed as

$$
S R F=1-\frac{\alpha_{d}}{\alpha_{u}}
$$

where $\alpha_{d}$ is the damaged ESP while $\alpha_{u}$ is the undamaged ESP.

To consider the uncertainties, the interval analysis is applied by specifying the input data upper and lower bounds. Then based on this input data, the output data upper and lower bounds can be formed. The interval at damage and undamaged condition of ESP $(\alpha)$ and DI $(H)$ can be expressed as:

$$
\begin{array}{ll}
{[\underline{\alpha}]=[\underline{H}]} & =\text { ESP value lower bound } \\
{[\bar{\alpha}]=[\bar{H}]} & =\text { ESP value upper bound }
\end{array}
$$




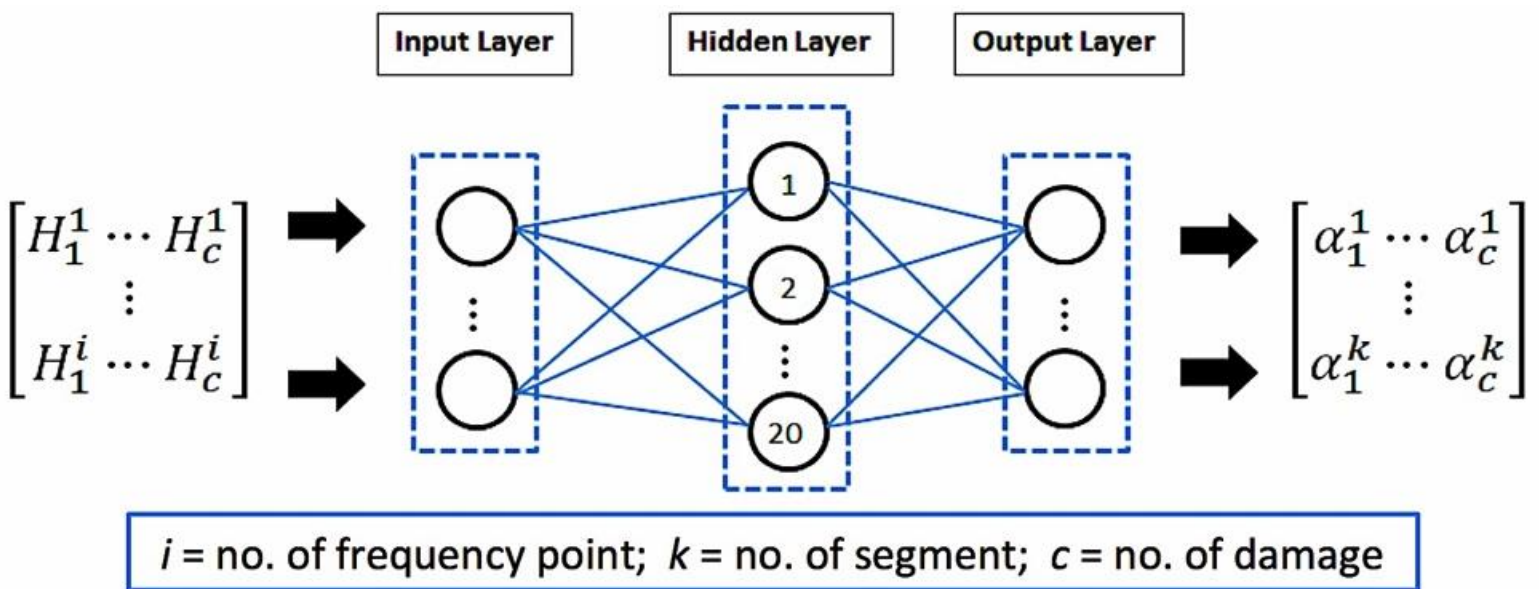

Fig. 1 - ANN architecture of multilayer perceptron

Therefore, every variable's interval bound for can be derived as follows:

$$
\begin{aligned}
& H_{c}^{I}=\left[\underline{H_{C}^{I}}, \overline{H_{c}^{I}}\right]=\left\{H_{c 1}^{I}, H_{c 2}^{I}, \cdots, H_{c i}^{I}\right\}^{T}, H_{c i}^{I}=\left[\underline{H_{c i}^{I}}, \overline{H_{c i}^{I}}\right] \\
& \alpha_{c}^{I}=\left[\underline{\alpha_{C}^{I}}, \overline{\alpha_{c}^{I}}\right]=\left\{\alpha_{c 1}^{I}, \alpha_{c 2}^{I}, \cdots, \alpha_{c k}^{I}\right\}^{T}, \alpha_{c k}^{I}=\left[\underline{\alpha_{c k}^{I}}, \overline{\alpha_{c k}^{I}}\right]
\end{aligned}
$$

where $c, i$, and $k$ is the number of damage cases, frequency points, and segments of the structures respectively. Then, the output middle value is signified as:

$$
\alpha^{c}=m\left(\alpha^{I}\right)=\frac{(\underline{\alpha}+\bar{\alpha})}{2}
$$

where $\underline{\alpha}$ is the lower bound and $\bar{\alpha}$ is the upper bounds of the exact output value.

The training and testing datasets of ANN will be formulated based on Eq. (4) - Eq. (8). Thus, two ANN of lower and upper bound formulations are computed as disclosed in Table 1.

Table 1 - Input and output variables for ANN training and testing phase

\begin{tabular}{cllc}
\hline Model & Training Input & Testing Input & Output \\
\hline ANN1 & $\underline{H_{c i}^{I r}}=H_{c i}^{I r}-H_{c i}^{I r}\left(\mathrm{~F}_{H}\right)$ & $\underline{H_{c i}^{I e}}=H_{c i}^{I e}-H_{c i}^{I e}\left(\mathrm{~F}_{H}\right)$ & $\underline{\alpha_{c k}}$ \\
\hline \multirow{2}{*}{ ANN2 } & $\overline{H_{c i}^{I r}}=H_{c i}^{I r}+H_{c i}^{I r}\left(\mathrm{~F}_{H}\right)$ & $\overline{H_{c i}^{I e}}=H_{c i}^{I e}+H_{c i}^{I e}\left(\mathrm{~F}_{H}\right)$ & $\overline{\alpha_{c k}}$
\end{tabular}

* Ir is the training phase and $I e$ is the testing phase interval while $\mathrm{F}$ is the uncertainty level for the input variables

By associating the vectors of the ESP interval bounds (outputs of ANN1 and ANN2), the PoDE can be measured as:

$$
\operatorname{PoDE}=\operatorname{possibility}\left(\alpha_{d k}<\alpha_{u k}\right)=\frac{A_{\text {damage }}}{A_{\text {total }}} \times 100 \%
$$

Using PoDE alone for damage detection will not provide the assessment of the damage since it will only deliver the location of the damage. Thus, DMI is employed to quantify the damage severity by multiplying the SRF by PoDE as:

$$
\beta_{D M I}=S R F \times P o D E
$$

\section{Example: Truss Bridge System}

Fig. 2 shows the fabricated and tested steel truss bridge structure with steel member type of 150UB14 that used to obtain the empirical FRF data. The dimension of the structure is stated in Fig. 3 where the length for each element is $250 \mathrm{~mm}$ and the whole truss size are $5000 \mathrm{~mm} \times 1000 \mathrm{~mm}$ x $866 \mathrm{~mm}$. The density of the truss, $\rho$ is equal to $7850 \mathrm{~kg} / \mathrm{m}^{2}$ 
and modulus of elasticity, $E$ is $2.0 \mathrm{e} 11 \mathrm{~Pa}$. The truss structure is modeled out of 185 beam elements. Every girder and web bar consists of four elements, and each diagram bar in the lateral direction consists of three elements. However, for the purpose the proposed approach, the truss elements is later grouped into 49 segments where for the web and girder bars, four elements are classified as one segment and for the diagram bars, three elements are classified as one segment. The segmentation number is stated in Fig. 3. To conduct the modal testing, one sensor is placed perpendicular towards the middle of segment 3 on the truss. The joints for each segment are rigid and the support system is fixed at four end of the lower main beam.

To validate the proposed method, three damage scenarios are forged as stated in Table 2 . The damage scenarios are introduced by piercing the member as presented in Fig. 4. The damage is organized by naming the cutting size for the main girder bar as M1 $(10 \times 75 \mathrm{~mm})$ and M2 $(20 \times 75 \mathrm{~mm})$, and for the web bar as W1 $(10 \times 75 \mathrm{~mm})$. The damage segments are selected based on the forces acting on its member. Damage M1 and W1 are in compression whereas M2 is in tension hence this condition will represent different damage on different member types. Thus, the ability of the proposed method can be intensified.

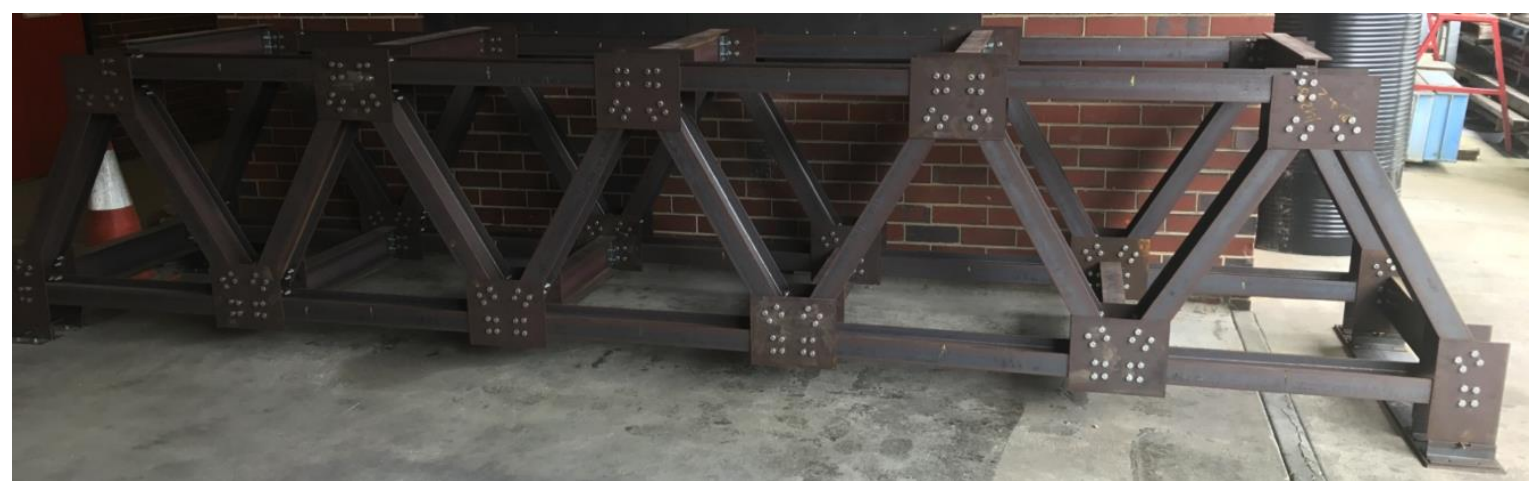

Fig. 2 - Laboratory scaled steel truss bridge structure

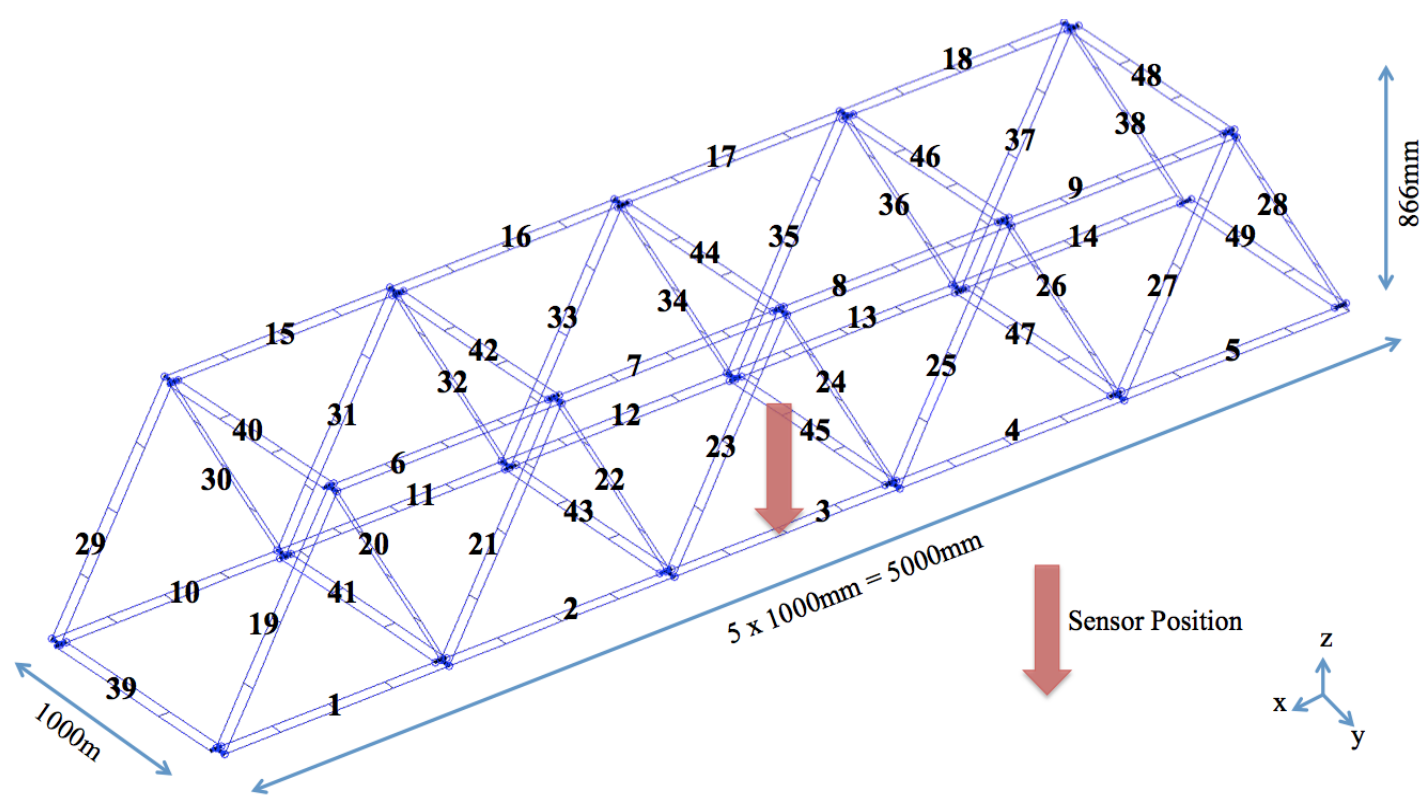

Fig. 3 - Segmentation, dimension, meshing and sensor position of the truss 
Table 2 - Damage scenario 1, 2 and 3 location and severity

\begin{tabular}{|c|c|c|c|c|c|c|c|}
\hline \multirow{2}{*}{ Segment } & \multicolumn{3}{|c|}{ Damage scenario } & \multirow{2}{*}{ Segment } & \multicolumn{3}{|c|}{ Damage scenario } \\
\hline & 1 & 2 & 3 & & 1 & 2 & 3 \\
\hline 1 & - & - & - & 26 & - & - & - \\
\hline 2 & - & - & - & 27 & - & - & - \\
\hline 3 & - & - & - & 28 & - & W1 & W1 \\
\hline 4 & - & - & M2 & 29 & - & - & - \\
\hline 5 & - & - & - & 30 & - & - & - \\
\hline 6 & - & - & - & 31 & - & - & - \\
\hline 7 & M1 & - & M1 & 32 & - & - & - \\
\hline 8 & - & - & - & 33 & - & - & - \\
\hline 9 & - & - & - & 34 & - & - & - \\
\hline 10 & - & - & - & 35 & - & - & - \\
\hline 11 & - & - & - & 36 & - & - & - \\
\hline 12 & - & - & - & 37 & - & - & - \\
\hline 13 & - & - & - & 38 & - & - & - \\
\hline 14 & - & - & - & 39 & - & - & - \\
\hline 15 & - & - & - & 40 & - & - & - \\
\hline 16 & - & - & - & 41 & - & - & - \\
\hline 17 & - & - & - & 42 & - & - & - \\
\hline 18 & - & - & - & 43 & - & - & - \\
\hline 19 & - & - & - & 44 & - & - & - \\
\hline 20 & - & - & - & 45 & - & - & - \\
\hline 21 & - & - & - & 46 & - & - & - \\
\hline 22 & - & - & - & 47 & - & - & - \\
\hline 23 & - & - & - & 48 & - & - & - \\
\hline 24 & - & - & - & 49 & - & - & - \\
\hline 25 & - & - & - & & & & \\
\hline
\end{tabular}
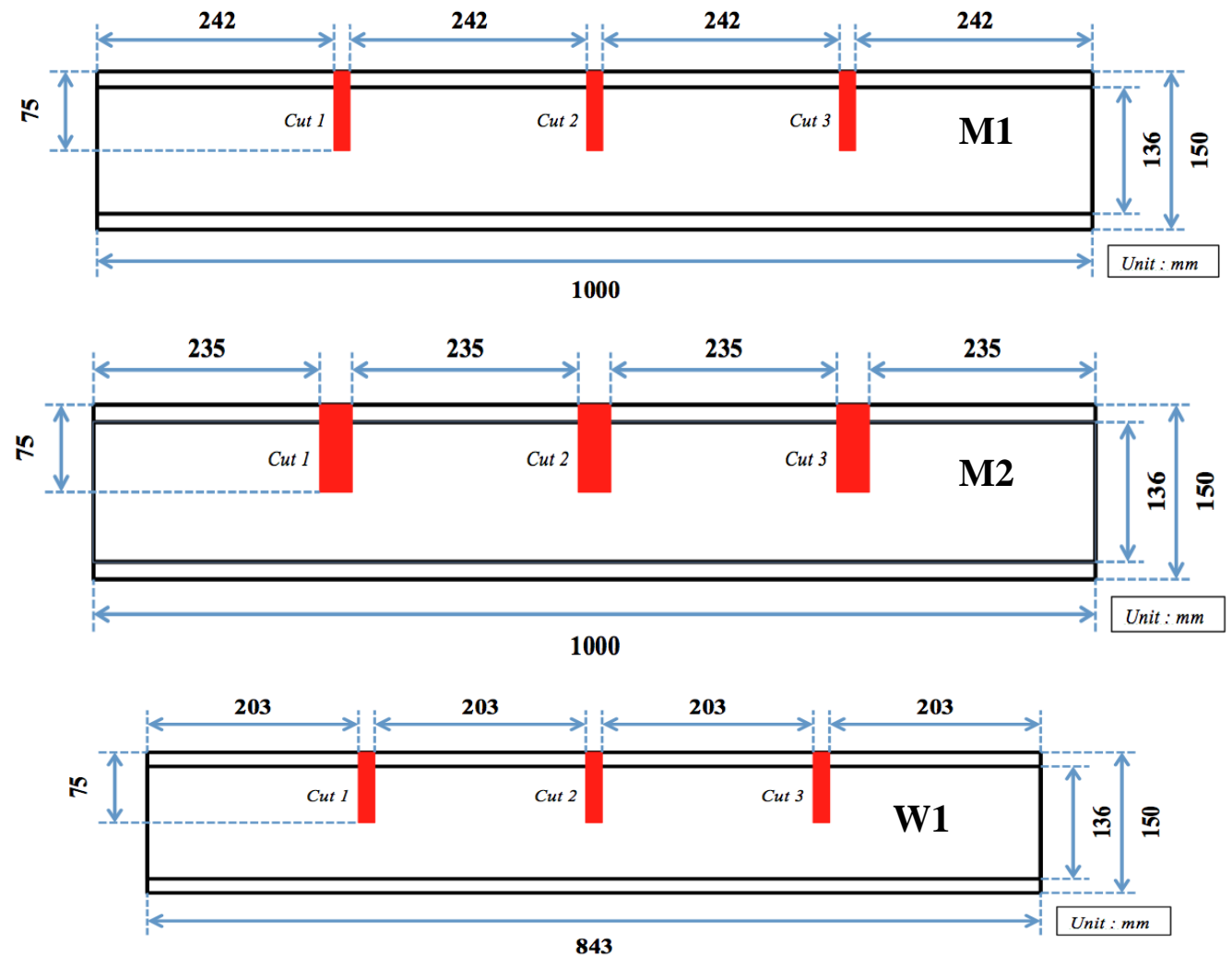

Fig. 4 - Executed damaged condition of truss members 


\section{Modal Testing and Damage Detection}

To obtain the empirical FRF data, modal testing procedure is conducted with one impact hammer and one sensor (184 ICP acceleration sensor). The impact hammer is excited at the horizontal beam of segment 43 and the sensor is placed at the main girder beam of segment 3 as shown in Fig. 3. Every damage scenario will have different modal test. As mentioned earlier, ANN training phase is conducted to establish the ANN baseline. The ANN is trained using 1200 random damage cases generated by the FE analysis of the truss structure. To avoid over fitting, early stopping method is implemented on the ANN model. Hence, the data is divided into the ratio of 6 to 4 for training and validation respectively. The frequency is measured from $0 \mathrm{~Hz}$ to $400 \mathrm{~Hz}$ with 512 points. The ANN input variables for the training phase are the PCA compressed FRF that are reduced from $(512 \times 1200)$ to $(90 \times 1200)$ matrix. Meanwhile for the testing phase, the ANN input variables FRF is compressed from $(6320 \times 3)$ to $(90 \times 3)$ matrix. Both phases are having the ESP values of each segment as the ANN output variables.

The proposed non-probabilistic method for damage detection is implemented once the ANN baseline model is established. The uncertainties level including data compression errors, modelling errors and measurement noise is assumed to be 5\%. Then, the PoDE and DMI values are measured based on the uncertainties assumption. Based on the proposed method, the PoDE values are shown in Table 3 and the DMI values are presented in Fig. 5, Fig. 6, and Fig. 7. The results of PoDE and DMI of the three damage scenarios demonstrate that the highest values of PoDE and DMI are at the exact location of executed damage. For instance, the damage segment for scenario 1 is at segment 7 and the PoDE value of the damage detection is $97.75 \%$ perceived at segment 7 where the exact executed damage is located. A similar trend is detected for scenarios 2 and 3, where the damage segments are segments 28, 4 and segments 7, 28 for scenario 2 and scenario 3 respectively. The PoDE values are observed at the highest above 95\% acquired at the exact performed damage locations. The damage severity for scenario 2 and scenario 3 are presented by the DMI values at the executed damage locations. These findings indicate that the proposed method is able to localize the damage as well as can provide the damage severity under the effect of uncertainties including the data compression errors, modelling errors and measurement noise in the empirical FRF data.

Table 3 - PoDE of damage scenario 1, 2 and 3

\begin{tabular}{|c|c|c|c|c|c|c|c|}
\hline \multirow{2}{*}{ Segment } & \multicolumn{3}{|c|}{ Damage scenario, PoDE (\%) } & \multirow{2}{*}{ Segment } & \multicolumn{3}{|c|}{ Damage scenario, PoDE (\%) } \\
\hline & 1 & 2 & 3 & & 1 & 2 & 3 \\
\hline 1 & 28.20 & 0.15 & 17.88 & 26 & 2.69 & 12.06 & 15.37 \\
\hline 2 & 4.24 & 0.00 & 2.29 & 27 & 11.68 & 18.95 & 28.10 \\
\hline 3 & 7.14 & 16.63 & 9.42 & 28 & 5.69 & 97.62 & 99.01 \\
\hline 4 & 12.16 & 13.91 & 99.85 & 29 & 2.80 & 18.46 & 2.71 \\
\hline 5 & 9.67 & 29.81 & 7.09 & 30 & 29.20 & 18.30 & 45.74 \\
\hline 6 & 11.81 & 26.65 & 9.59 & 31 & 43.17 & 5.18 & 0.00 \\
\hline 7 & 97.75 & 7.81 & 100.00 & 32 & 1.55 & 9.04 & 1.81 \\
\hline 8 & 32.24 & 2.80 & 3.85 & 33 & 0.00 & 11.71 & 0.00 \\
\hline 9 & 14.99 & 0.00 & 3.36 & 34 & 16.72 & 6.53 & 3.77 \\
\hline 10 & 35.96 & 7.11 & 6.88 & 35 & 10.62 & 0.00 & 0.00 \\
\hline 11 & 51.86 & 3.26 & 31.96 & 36 & 37.24 & 0.00 & 24.07 \\
\hline 12 & 0.00 & 16.12 & 3.09 & 37 & 19.81 & 23.02 & 7.61 \\
\hline 13 & 14.90 & 0.00 & 5.44 & 38 & 8.97 & 4.39 & 28.91 \\
\hline 14 & 0.00 & 26.92 & 40.76 & 39 & 3.95 & 28.41 & 7.18 \\
\hline 15 & 5.38 & 19.04 & 0.00 & 40 & 0.00 & 10.78 & 9.51 \\
\hline 16 & 43.24 & 6.89 & 0.00 & 41 & 0.00 & 0.00 & 6.80 \\
\hline 17 & 0.00 & 6.07 & 0.00 & 42 & 5.27 & 0.00 & 25.48 \\
\hline 18 & 8.35 & 0.00 & 3.13 & 43 & 3.79 & 0.00 & 22.40 \\
\hline 19 & 13.32 & 15.64 & 32.51 & 44 & 26.91 & 6.07 & 6.78 \\
\hline 20 & 17.34 & 0.00 & 10.56 & 45 & 12.28 & 19.44 & 0.00 \\
\hline 21 & 39.09 & 4.48 & 1.10 & 46 & 14.79 & 2.25 & 34.45 \\
\hline 22 & 0.00 & 17.63 & 9.00 & 47 & 0.00 & 12.53 & 0.00 \\
\hline 23 & 0.00 & 19.09 & 2.35 & 48 & 11.77 & 11.27 & 0.00 \\
\hline 24 & 6.05 & 2.83 & 9.08 & 49 & 0.00 & 16.15 & 0.67 \\
\hline 25 & 39.93 & 8.20 & 26.65 & & & & \\
\hline
\end{tabular}




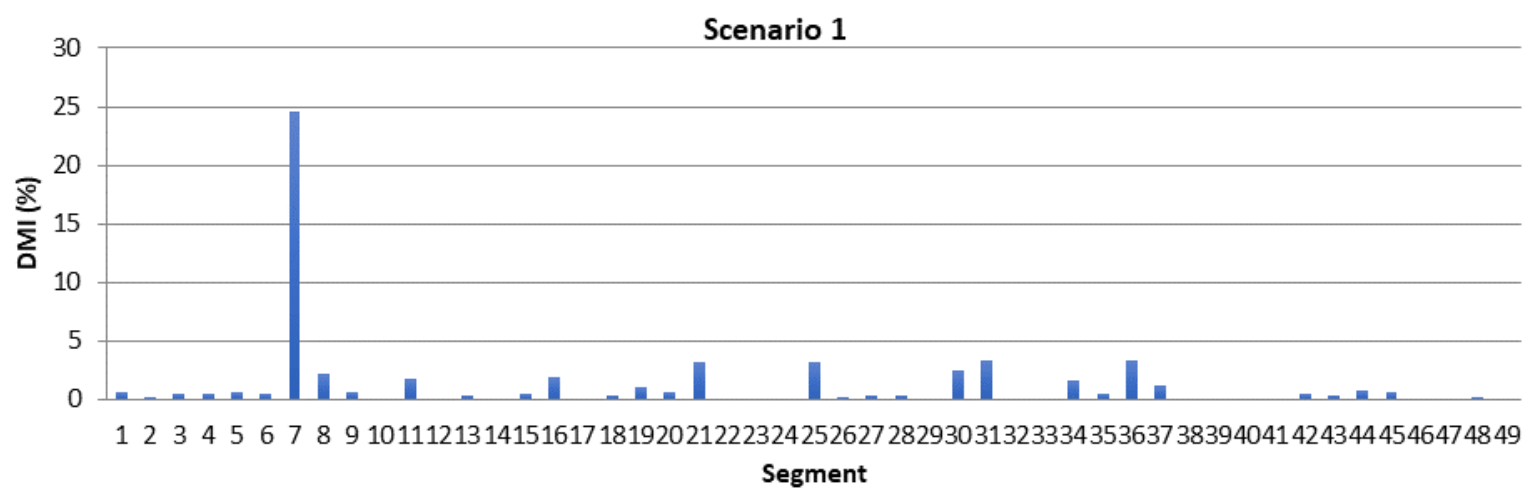

Fig. 5 - Scenario 1 damage measure index (DMI)

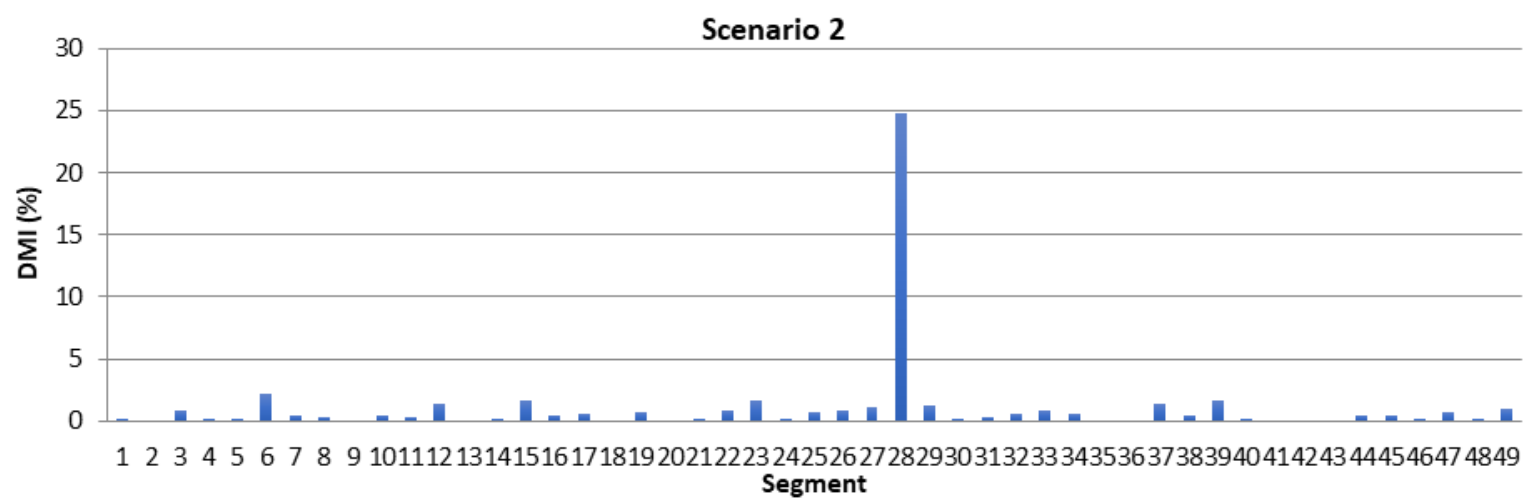

Fig. 6 - Scenario 2 damage measure index (DMI)

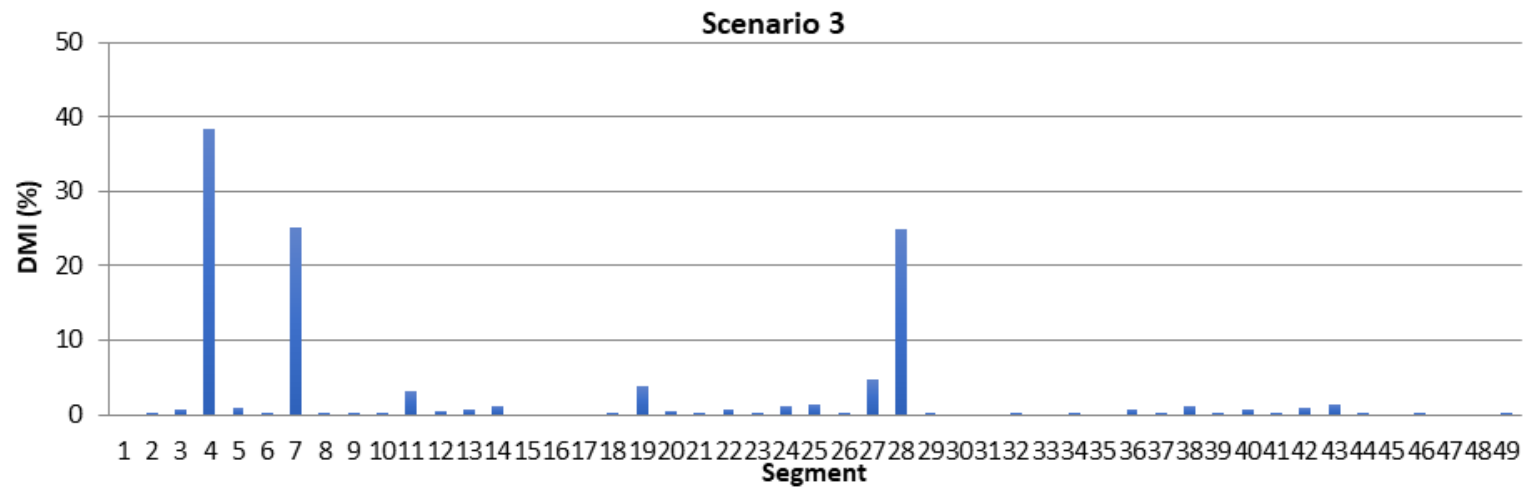

Fig. 7 - Scenario 3 damage measure index (DMI)

\section{Conclusion}

This study proposed a merger of non-probabilistic analysis with ANN approach to consider the uncertainties effect on compressed empirical FRF data in vibration-based damage detection. The non-probabilistic analysis applied the concept of interval analysis approach by considering the uncertainties with the lower and upper bounds intervals. The damage location and severity are defined by measuring PoDE and DMI respectively. The empirical FRF data is obtained by modal testing procedure conducted on experimental model of a steel truss bridge. To compress the large size of the empirical FRF data, PCA is formulated to extract the important features of the structural damage condition. Three damage cases are executed to verify the proposed method. Based on the results of PoDE and DMI, the proposed method is efficient in considering the uncertainties effect on empirical FRF data under short computational time. The highest PoDEs above 95\% are observed at the exact executed damage location and the damage severity is quantified at the exact performed damage location using DMI formulation. 


\section{Acknowledgments}

The authors would like to express their appreciation to the Ministry of Higher Education, Malaysia, and Universiti Teknologi Malaysia (UTM) for their financial support through the HICOE Grant vot 4J224 and Transdisciplinary Research Grant vot 07G46.

\section{References}

[1] Vazirizade S. M., Bakhshi A. \& Bahar O. (2018). Structural damage detection using ensemble empirical mode decomposition. Hilbert Transform and Artificial Neural Networks. arXiv:1812.02724 [eess.SP]

[2] Tan Z. X., Thambiratnam D. P., Chan T. H. T. \& Razak H. A. (2017). Detecting damage in steel beams using modal strain energy based damage index and artificial neural network. Engineering Failure Analysis, 79, 253-262

[3] Rao P. S. \& Mahendra N. (2018). Vibration based damage identification method for cantilever beam using artificial neural network. Lecture Notes in Civil Engineering Springer, 5, 85-93

[4] Nguyen V., Dackermann U., Li J., Makki Alamdari M., Mustapha S., Runcie P. \& Ye L. (2015). Damage identification of a concrete arch beam based on frequency response functions and artificial neural networks. Electronic Journal of Structural Engineering, 14(1), 75-84

[5] Bai F., Wang X., Liu Y., Liu X., Xiang Y. \& Liu Y. (2016). Measurement-based frequency dynamic response estimation using geometric template matching and recurrent artificial neural network. CSEE Journal of Power and Energy Systems, 2(3), 10-18

[6] Tamuz O., Mazeh T. \& Zucker S. (2005). Correcting systematic effects in a large set of photometric light curves. Monthly Notices of the Royal Astronomical Society, 356(4), 1466-1470

[7] Sharpe J. \& Fieller N. (2016). Uncertainty in functional principal component analysis. Journal of Applied Statistics, 43(12), 2295-2309

[8] Wang X., Xia Y., Zhou X. \&Yang C. (2014). Structural damage measure index based on non-probabilistic reliability model. Journal of Sound and Vibration, 333(5), 1344-1355

[9] Xia Y., Wang X. J. \& Zhou X. Q. (2013). Damage Detection based on non-probabilistic reliability model. Proceeding 15th Asia Pacific Vibration Conference 2013, Jeju, pp. 1157-1162

[10] Padil K. H., Bakhary N. \& Hao H. (2017). The use of a non-probabilistic artificial neural network to consider uncertainties in vibration-based-damage detection. Mechanical Systems and Signal Processing, 83, 194-209

[11] Abdulkareem M., Bakhary N., Vafaei M., Noor N. M. \& Padil K. H. (2018). Non-probabilistic wavelet method to consider uncertainties in structural damage detection. Journal of Sound and Vibration, 433, 77-98

[12] Pradhan S. \& Modak S. (2012). Normal response function method for mass and stiffness matrix updating using complex FRFs. Mechanical Systems and Signal Processing, 32, 232-250 